\title{
A Novel Autophagy-Related IncRNA Prognostic Signature Associated with Immune Microenvironment and Survival Outcomes of Gastric Cancer Patients
}

\author{
Di Chen \\ Mengmeng Wang \\ Yushuang $\mathrm{Xu}$ \\ Xin Jiang \\ Lina Xiong \\ Li Zhang \\ Honglu Yu \\ Zhifan Xiong
}

Department of Gastroenterology, Liyuan Hospital, Tongji Medical College,

Huazhong University of Science and

Technology, Wuhan, People's Republic of China
Correspondence: Zhifan Xiong Department of Gastroenterology, Liyuan Hospital, Tongii Medical College,

Huazhong University of Science and

Technology, Wuhan, 43006I, People's

Republic of China

Tel +86 I35I7281937

Email xiongzhifan@I26.com
Purpose: Autophagy plays a crucial role in the initiation and progression of gastric cancer (GC). However, the role of autophagy-related lncRNAs in GC remains unknown. This study aimed to investigate the prognostic value of the autophagy-related lncRNA signature and its role in the tumor immune microenvironment (TIME) of GC.

Methods: RNA-sequencing (RNA-seq) and clinical data of GC patients were extracted from The Cancer Genome Atlas (TCGA) database. Univariate and multivariate Cox regression analyses were performed to identify the autophagy-related lncRNA prognostic signature which was validated in the test set and entire set. The survival and predictive performance were analyzed based on the Kaplan-Meier and ROC curves. Furthermore, the CIBERSORT algorithm was applied to explore the relationship between this signature and the immune cell infiltration. To elucidate the potential functions of autophagy-related lncRNAs in GC, we constructed the IncRNA-mRNA co-expression network and performed enrichment analysis. Principal component analysis (PCA) and Gene Set Enrichment Analysis (GSEA) were further performed to compare the different statuses between the high-risk and low-risk groups.

Results: We identified 5 autophagy-related lncRNAs (AL355574.1, AC010768.2, AP000695.2, AC087286.2, and HAGLR) to construct a prognostic signature. This signature could be an independent prognostic indicator for GC patients and had a higher prediction efficiency than other clinicopathological parameters. Furthermore, patients in the high-risk score group had a stronger immunosuppressive microenvironment than the low-risk group. The enrichment analysis for mRNAs co-expressed with these lncRNAs indicated that autophagy-related signaling pathways were remarkably enriched. PCA and GSEA further revealed different autophagy and immune statuses in the high- and low-risk groups.

Conclusion: The 5 autophagy-related lncRNA signature has significant clinical implications in prognosis prediction of GC. Meanwhile, our study elucidates the critical role of the autophagy-related lncRNA signature in the TIME of GC.

Keywords: gastric cancer, long non-coding RNAs, autophagy, prognostic signature, tumor immune microenvironment

\section{Introduction}

Gastric cancer (GC) is a globally crucial disease. With 768,793 death cases globally in 2020, GC is the fourth leading cause of cancer death. ${ }^{1}$ Although great progress has been made in risk factors, early diagnosis, and treatments, the prognosis of GC patients is still pessimistic. $^{2-4}$ The prognosis of GC is correlated with pathological 
stage, biological behavior, tissue type, and treatment. ${ }^{5}$ TNM staging has been one of the primary methods to evaluate the prognosis of patients with GC. ${ }^{6}$ Nevertheless, the current TNM staging system cannot include all disease information of patients and has limited power to predict the prognosis of GC. Therefore, new strategies are urgently needed to improve survival outcome prediction and optimize clinical treatment decisions.

Autophagy is a critical cellular process to sustain metabolism and homeostasis by capturing and degrading intracellular components such as proteins and organelles. ${ }^{7,8}$ Apart from its role in normal physiology, autophagy also plays an essential role in various pathological processes such as neurodegenerative disorders, cancer, and inflammation. ${ }^{7,9-11}$ Recently, the role of autophagy in cancer has attracted more and more attention from the cancer research community. A growing number of studies have revealed that autophagy participates in GC progression and prognosis. $^{12-14}$ Thus, investigation of autophagyrelated molecules is crucial for both theoretical basis and clinical practice. Long non-coding RNAs (lncRNAs) are a class of transcripts that exceed $200 \mathrm{nt}$ in length and have no apparent protein-coding capacity. ${ }^{15,16}$ In recent years, accumulating evidence has revealed that dysregulated lncRNAs are extensively involved in various tumors, including GC. ${ }^{17-19}$ Furthermore, lncRNAs have been found to affect the progression and prognosis of tumors through modulating autophagy. For example, Wu et al demonstrated that upregulated SNHG11 is correlated with poor prognosis of GC patients. Functionally, SNHG11 facilitates the proliferation, migration, and invasion of GC cells by activating oncogenic autophagy. ${ }^{20}$ HAGLROS is also identified as an oncogenic lncRNA, which promotes GC progression via mTOR pathway mediated autophagy suppression. ${ }^{21}$ However, these studies investigated the association between autophagy-related lncRNAs and GC prognosis; few studies explored the role of autophagy-related lncRNA signature in the tumor immune microenvironment (TIME).

In the present study, we aimed to assess autophagyrelated lncRNA signature with the prognosis and TIME in GC. We analyzed IncRNA expression profiles of GC patients from The Cancer Genome Atlas (TCGA) and explored autophagy-related lncRNAs with prognostic value. Then, we identified a 5 autophagy-related lncRNA prognostic signature. This signature could be a powerful indicator of GC patinets' clinical outcome. Furthermore, based on the autophagy-related signature, the relationship between risk score and infiltrating immune cells was analyzed to explore the effect of autophagy-related lncRNAs in the TIME.

\section{Materials and Methods}

Data Acquisition and Identification of Autophagy-Related IncRNAs

A detailed workflow of the study is shown in Figure 1. The RNA-sequencing (RNA-seq) data of 339 GC and 30 nontumor samples were downloaded from TCGA. All patients with the disease types of "adenomas or adenocarcinomas" were included in this study. LncRNAs and mRNAs were further classified based on the gene annotations in the GENCODE project. The corresponding patients' clinicopathological parameters, such as grade, survival information, and tumor stage, were also derived from TCGA. Patients with incomplete RNA-seq data and survival information were excluded, and the clinicopathological characteristics of 339 GC patients are listed in Table S1. All data in our study were available from the TCGA database. According to the ethics committee of Liyuan Hospital of Tongji Medical College of Huazhong University of Science and Technology guidelines, the data from the TCGA database is publicly accessible, and no ethical approval was considered necessary. Furthermore, a total of 232 autophagy-related genes (ATGs) were downloaded from the Human Autophagy Database (HADb), which contains a list of genes involved in the autophagy process reported in the literature. To evaluate the correlation of lncRNAs and ATGs, Pearson correlation analysis was performed in the statistical software $\mathrm{R}$ (version 4.0.2). Based on the correlation coefficient $(|\mathrm{R}|>0.3$ and $P<$ $0.001)$, the autophagy-related IncRNAs were determined and used for further analysis.

\section{Construction of the Prognostic Signature}

The R package "caret" was utilized to randomly separate GC samples into the train set (170 samples) and the test set (169 samples). To explore the prognostic value of autophagy-related lncRNAs, the R package "survival" was used to perform univariate Cox regression analysis. Those autophagy-related lncRNAs significantly associated with survival $(P<0.05)$ were selected as candidate autophagyrelated lncRNAs for further research. A hazard ratio (HR) value was used to identify protective lncRNAs $(\mathrm{HR}<1)$ and deleterious lncRNAs (HR $>1$ ). Subsequently, multivariate Cox regression analysis was performed to evaluate 


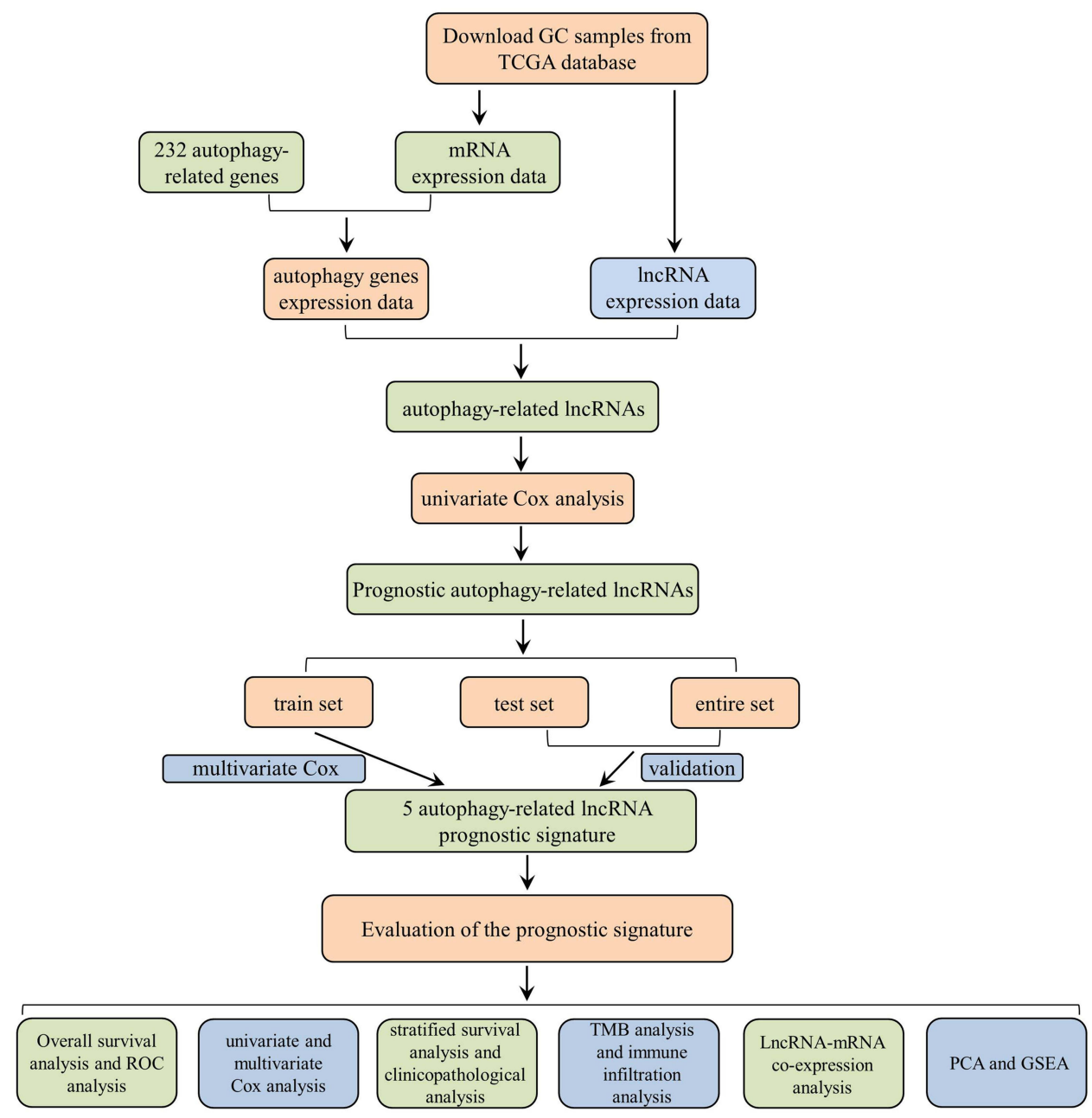

Figure I The flow chart of this study.

whether candidate autophagy-related lncRNAs could be used as independent prognostic predictors. Based on the Akaike information criterion, we constructed the optimal autophagy-related lncRNA prognostic signature. We further calculated the risk score for each patient according to the following algorithm:

Risk score $=($ coefficient $\operatorname{lncRNA} 1 \times$ expression $\operatorname{lncRNA} 1)+$ (coefficient $\operatorname{lncRNA2} \times$ expression $\operatorname{lncRNA2})+\ldots+($ coefficient $\operatorname{lncRNAn} \times$ expression lncRNAn).

\section{Evaluation of the Prognostic Signature}

To investigate the autophagy-related lncRNA signature in predicting patients' survival, GC patients in the train set were classified into the low-risk and high-risk groups based on the median risk score. The overall survival (OS) of patients in two groups was compared using the Kaplan-Meier analysis and a Log rank test. Furthermore, to assess whether the risk score was an independent factor for GC patients' survival, we performed univariate and multivariate Cox regression analyses using the R package "survival". The receiver-operating characteristic (ROC) curves were used to assess the predictive performance of the prognostic signature by $\mathrm{R}$ language loaded with package "survivalROC". The test set and entire set were utilized to validate the prognostic signature. But beyond all that, stratified survival analysis was applied to detect the prognostic signature's accuracy in different subgroups. $P<0.05$ was set as the threshold.

\section{Clinicopathological Characteristics Analysis, Tumor Mutational Burden (TMB) Analysis, and Immune Microenvironment Assessment}

We evaluated the association between the risk score and clinicopathological characteristics, including grade, T, N, M, and tumor stage. In addition, we downloaded the tumor mutational 
data from TCGA and calculated TMB with the tumor-specific mutation genes. After that, we analyzed the top 30 mutational genes in the low-risk and high-risk groups using the R package "maftools". To explore the immune infiltrating landscape of the TME in different risk groups, we performed CIBERSORT algorithms and Single-sample Gene Set Enrichment Analysis (ssGSEA). $P<0.05$ was set as the threshold.

\section{LncRNA-mRNA Co-Expression Analysis} and Functional Enrichment Analysis

Pearson correlation analysis was carried out to evaluate the relationship between the autophagy-related lncRNAs and their target mRNAs. $|\mathrm{R}|>0.3$ and $p<0.001$ were set as the cutoff criterion. Then, we constructed the IncRNA-mRNA coexpression network using the Cytoscape software. Furthermore, gene ontology (GO) and Kyoto Encyclopedia of Genes and Genomes (KEGG) enrichment analysis for mRNAs co-expressed with autophagy-related lncRNAs were performed by the packages "clusterProfiler", "ggplot2", and "enrichplot" in R.

\section{Principal Component Analysis (PCA) and Gene Set Enrichment Analysis (GSEA)}

PCA was performed to analyze the differences and similarities among grouped samples based on the whole-genome expression profiles, autophagy-related lncRNA set, and autophagyrelated lncRNA prognostic signature. The analyses were implemented using the R packages "scatterplot $3 d$ " and "limma". Furthermore, we explored whether the autophagy pathway was enriched in high-risk groups. GSEA was applied to the low- and high-risk groups of the autophagy-related IncRNA prognostic signature using GSEA software version 3.0. $P<0.05$ was regarded as the threshold.

\section{Statistical Analyses}

Statistical analyses were carried out using Perl version 5.30.1 and $\mathrm{R}$ version 4.0.2. All statistical methods and appropriate $\mathrm{R}$ packages were described throughout the study. $P$-value $<0.05$ was considered statistically significant.

\section{Results}

\section{Identification of Autophagy-Related IncRNAs with Prognostic Significance in the Train Set}

A total of 7009 lncRNAs were extracted from the TCGA database, and a list of 232 ATGs was obtained from the
$\mathrm{HADb}$. Then, 852 autophagy-related lncRNAs were identified based on the Pearson correlation analysis $(|\mathrm{R}|>0.3$ and $P<$ 0.001). Among them, 17 autophagy-related lncRNAs were related to the GC patients' survival by univariate Cox regression analysis, including 11 deleterious lncRNAs $(H R>1)$ and 6 protective lncRNAs $(\mathrm{HR}<1)$ (Figure 2). Furthermore, multivariate Cox analysis filtered out 5 autophagy-related lncRNAs (AL355574.1, AC010768.2, AP000695.2, AC087286.2, and HAGLR) in the train set to construct the optimal prognostic signature (Table 1). The following algorithm was utilized to calculate the risk score for each sample according to the 5 autophagy-related lncRNA prognostic signature: risk score $=$ $(-0.256 \times$ AL355574.1 expression $)+(-0.488 \times$ AC010768.2 expression $)+(0.176 \times$ AP000695.2 expression $)+(0.376 \times$ AC087286.2 expression $)+(0.114 \times$ HAGLR expression $)$. The risk score of $339 \mathrm{GC}$ samples are listed in Table S2.

To explore the significance of autophagy-related lncRNA signature in GC patients' prognosis, we divided all samples into the low-risk and high-risk groups. We ranked all GC patients and analyzed their distributions according to the lncRNA prognostic signature-based risk scores (Figure 3A). The scatter dot plot of survival status revealed that GC patients in the high-risk group had a shorter survival time than those in the low-risk group (Figure 3B). The heatmap was drawn to compare expression levels of the 5 autophagy-related IncRNAs in the high- and low-risk groups. GC patients with the low-risk score expressed higher levels of protective IncRNAs (AL355574.1 and AC010768.2), while those with the high-risk score expressed higher levels of deleterious IncRNAs (AC087286.2, AP000695.2, and HAGLR) (Figure 3C). Furthermore, the Kaplan-Meier survival curve indicated that the survival rate of GC patients in the high-risk group was remarkably decreased compared to the low-risk group ( $P=3.467 \mathrm{e}-08$ ) (Figure $3 \mathrm{D})$. ROC curve analysis showed that the area under the curve (AUC) values was $0.801,0.808,0.807$, respectively, for predicting 1-, 2-, and 3year survival rates (Figure 3E).

\section{Validation of the Autophagy-Related IncRNA Prognostic Signature in the Test Set and Entire Set}

To validate the autophagy-related lncRNA signature, risk scores were calculated in the test set and entire set with the same algorithm of the train set. According to the cutoff value of the train set, GC patients in the test set and the entire set were also respectively divided into the low- and high-risk groups. Similar to the train set, patients in the 


$\begin{array}{lrr} & \text { pvalue } & \text { Hazard ratio } \\ \text { AC244153.1 } & 0.031 & 1.112(1.009-1.225) \\ \text { AL355574.1 } & 0.004 & 0.801(0.690-0.931) \\ \text { MSC-AS1 } & 0.003 & 1.571(1.164-2.122) \\ \text { AC037198.1 } & 0.004 & 1.342(1.096-1.643) \\ \text { AP001528.2 } & 0.005 & 1.459(1.124-1.894) \\ \text { AC010768.2 } & 0.048 & 0.666(0.446-0.996) \\ \text { AC009948.1 } & 0.024 & 1.202(1.024-1.411) \\ \text { TNFRSF10A-AS1 } & 0.034 & 0.908(0.830-0.993) \\ \text { AP000695.2 } & 0.002 & 1.267(1.089-1.475) \\ \text { IPO5P1 } & 0.043 & 0.829(0.691-0.994) \\ \text { MAGI2-AS3 } & 0.013 & 1.242(1.046-1.475) \\ \text { AC004596.1 } & 0.037 & 0.680(0.474-0.976) \\ \text { AC087286.2 } & 0.002 & 1.510(1.169-1.951) \\ \text { AC104083.1 } & 0.023 & 1.023(1.003-1.044) \\ \text { UBL7-AS1 } & 0.008 & 0.596(0.405-0.876) \\ \text { AC005586.1 } & 0.004 & 0.774(0.652-0.920) \\ \text { HAGLR } & 0.008 & 1.082(1.021-1.147)\end{array}$

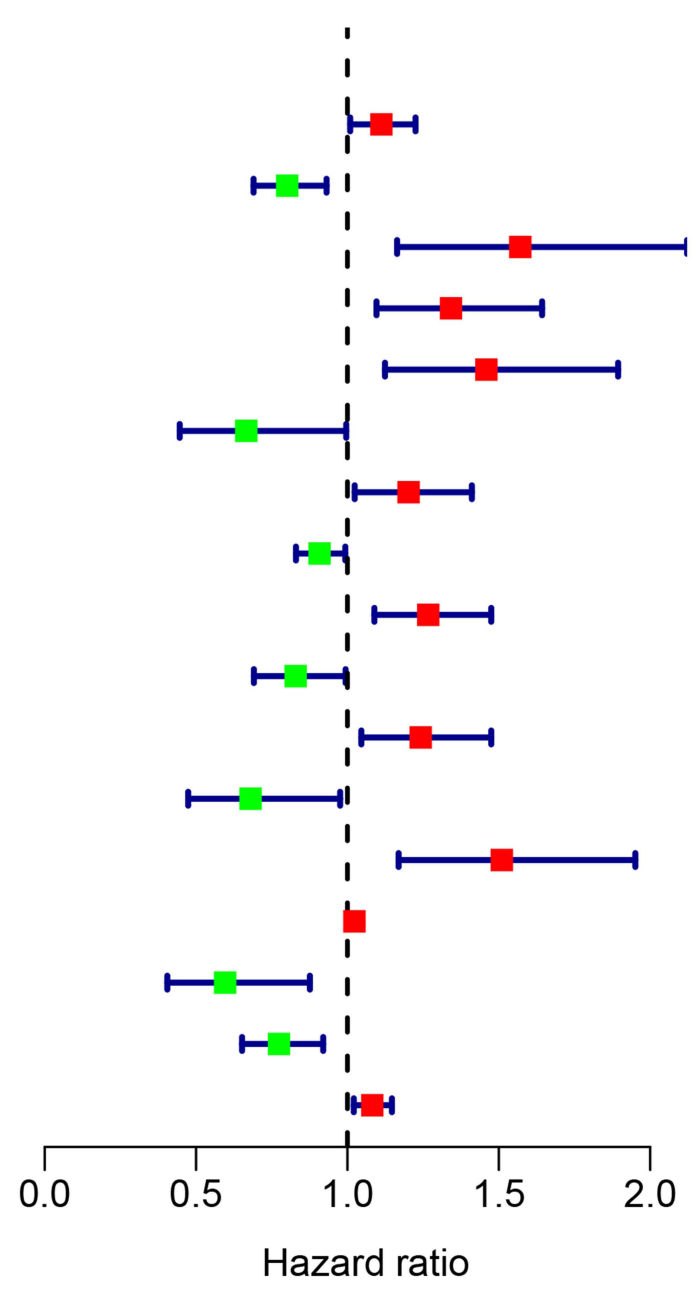

Figure 2 Identification of autophagy-related IncRNAs with significant prognostic value in GC.

high-risk group of the test set and the entire set had a higher probability of death than those in the low-risk group (Figure 4A-F). Furthermore, the Kaplan-Meier survival curve indicated that GC patients in the high-risk group of the test set $(P=3.213 \mathrm{e}-02)$ and the entire set $(P=4.798 \mathrm{e}-08)$ had a poorer prognosis than the low-risk group (Figure 4G and I). In the test set, the AUC values of $1-, 2-$, and 3-year survival rates were $0.616,0.635,0.651$, respectively (Figure 4H). Similarly, in the entire set, the results showed that the AUC values of 1-, 2-, and 3-year survival rates were $0.711,0.729$, and 0.743 , respectively (Figure 4J).

\section{Independence of the Autophagy-Related IncRNA Signature for GC Patients}

Univariate and multivariate Cox regression analyses were performed to explore whether the autophagyrelated lncRNA prognostic signature was an independent prognostic factor. In the train set, the result of univariate analysis exhibited that tumor stage $(P<$

Table I The 5 Autophagy-Related IncRNA Prognostic Signature

\begin{tabular}{|l|l|l|l|l|l|}
\hline LncRNA & Coef & HR & HR.95L & HR.95H & P-value \\
\hline AL355574.1 & -0.256 & 0.774 & 0.662 & 0.907 & 0.001 \\
AC010768.2 & -0.488 & 0.614 & 0.400 & 0.942 & 0.026 \\
AP000695.2 & 0.177 & 1.193 & 1.006 & 1.416 & 0.043 \\
AC087286.2 & 0.376 & 1.457 & 1.108 & 1.916 & 0.007 \\
HAGLR & 0.115 & 1.122 & 1.050 & 1.199 & 0.001 \\
\hline
\end{tabular}


A

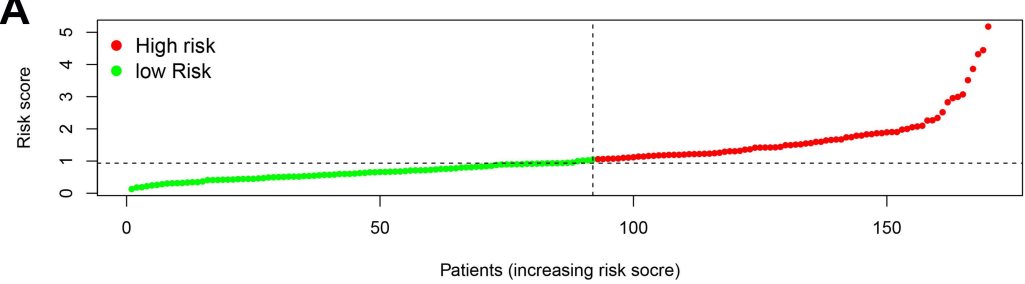

B

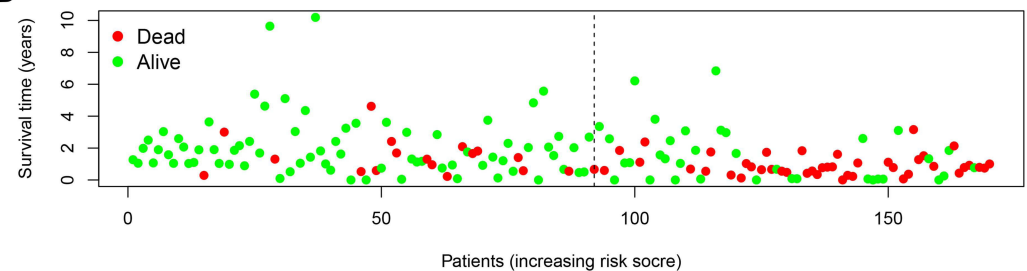

C

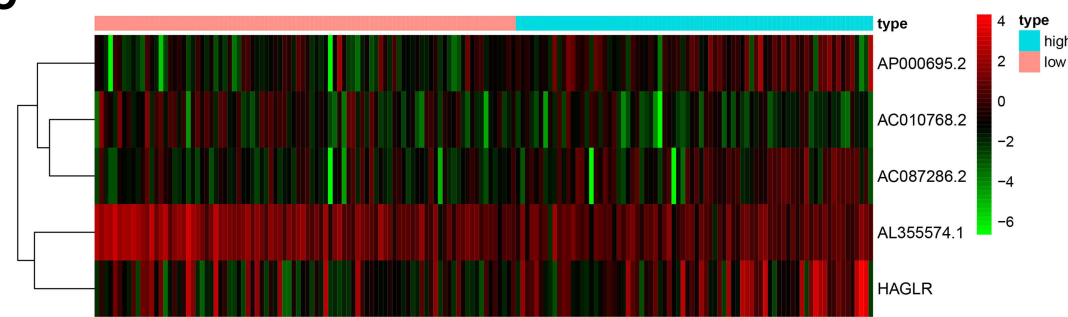

D

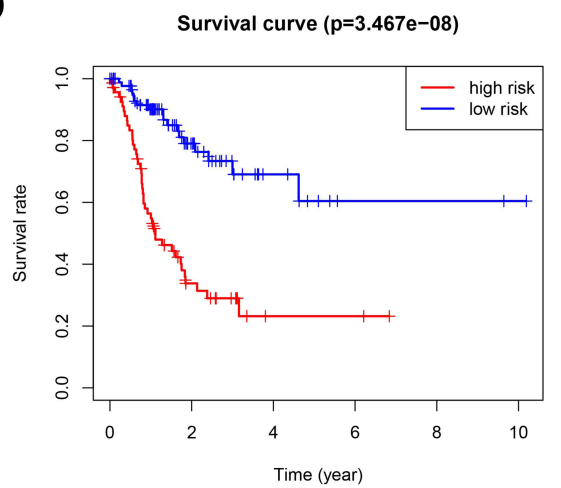

E

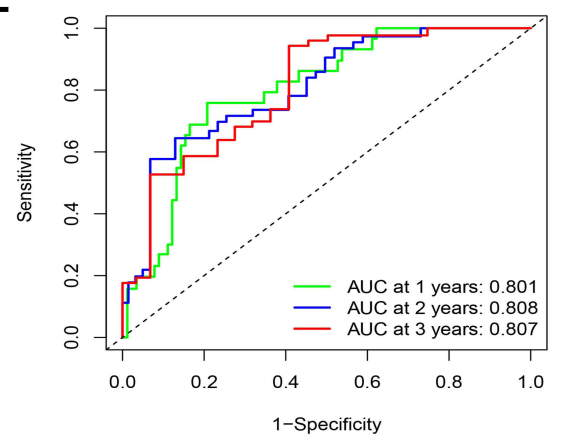

Figure 3 The prognostic value of the 5 autophagy-related IncRNAs signature in the train set. (A) The number of patients in the high-risk and low-risk groups ranked by the risk score. (B) The scatter dot plot of GC patients' survival status. (C) The heatmap of the 5 autophagy-related IncRNAs expression. (D) Kaplan-Meier survival analysis for patients between the high-risk and low-risk groups. (E) The ROC for the autophagy-related IncRNAs signature.

$0.001)$, N stage $(P<0.001)$, and risk score $(P<0.001)$ were significantly correlated with OS (Figure 5A). Multivariate analysis suggested that risk score $(P=$ 0.003) was an independent prognostic factor for OS (Figure 5D). ROC curve analysis showed that the AUC of risk score was 0.819 , which was higher than other clinicopathological parameters (Figure 5G). In the test set, result of univariate analysis showed that tumor stage $(P=0.037)$, T stage $(P=0.038)$ and risk score $(P<0.001)$ were significantly correlated with OS (Figure 5B). Multivariate analysis indicated that risk score $(P=0.001)$ was also an independent prognostic factor for OS (Figure 5E). Furthermore, ROC curve analysis showed that the AUC of risk score was 0.623, which was higher than other clinicopathological parameters (Figure 5H). Similarly, in the entire set, age $(P<0.001)$ and risk score $(P<0.001)$ were independent prognostic factors for OS (Figure $5 \mathrm{C}$ and $\mathrm{F}$ ). The AUC of risk score was 0.720 , which was also higher than other clinicopathological parameters (Figure 5I).
Correlation of Clinicopathological Characteristics and the AutophagyRelated IncRNA Prognostic Signature

We also evaluated the relationship between the autophagyrelated lncRNA prognostic signature and the clinicopathological features of GC patients in the entire TCGA. The heatmap exhibited that the risk score from the prognostic signature was significantly associated with tumor stage ( $P$ $<0.01)$ and survival state $(P<0.001)$ (Figure 6$)$. The results testified that the autophagy-related lncRNA signature might play a crucial role in GC initiation and progression.

We further performed the stratification analysis in the entire set to demonstrate the extensive usage of the prognostic signature. The patients were grouped based on gender (male and female), age $(<65$ and $\geq 65$ ), grade (low grade and high grade), tumor stage (stage I+II and stages III $+\mathrm{IV}), \mathrm{T}$ stage $(\mathrm{T} 1+2$ and $\mathrm{T} 3+4), \mathrm{N}$ stage $(\mathrm{N} 0+1$ and $\mathrm{N} 2+3$ ) and $\mathrm{M}$ stage (M0 and M1). As shown in Figure 7, survival analysis revealed that the survival of 

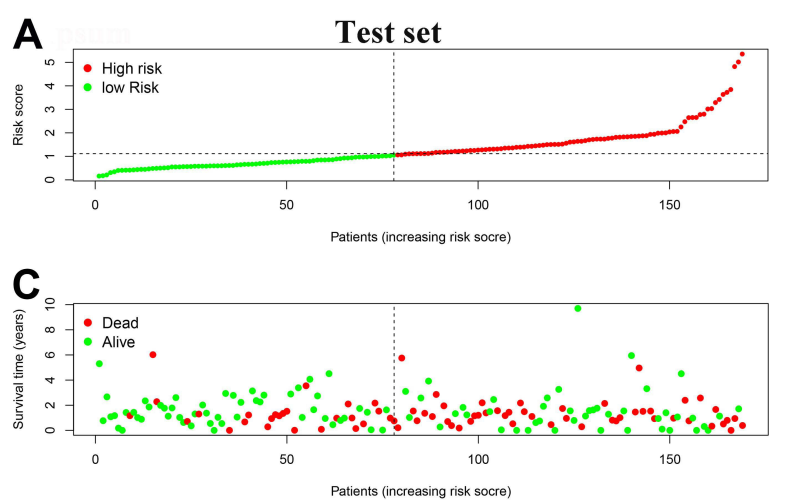

E

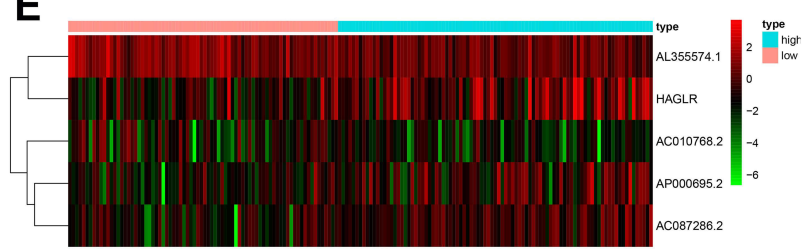

G

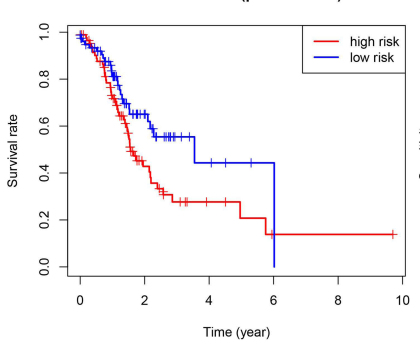

H

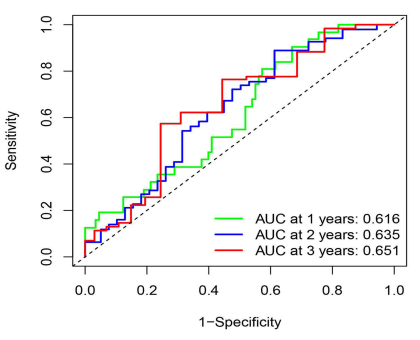

B

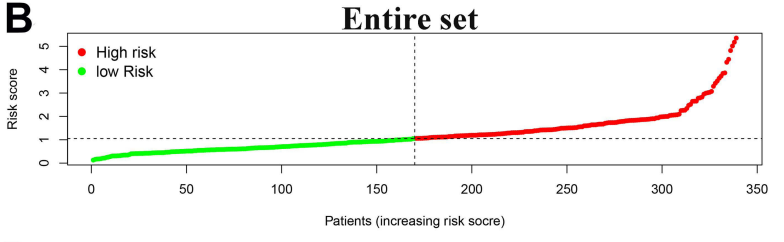

D

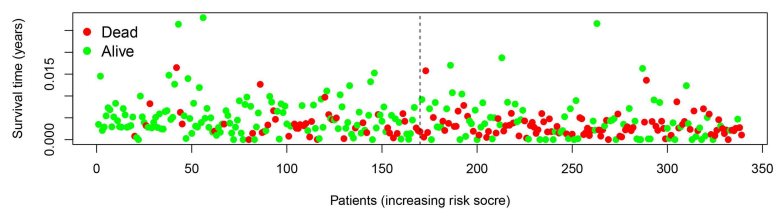

$\mathbf{F}$

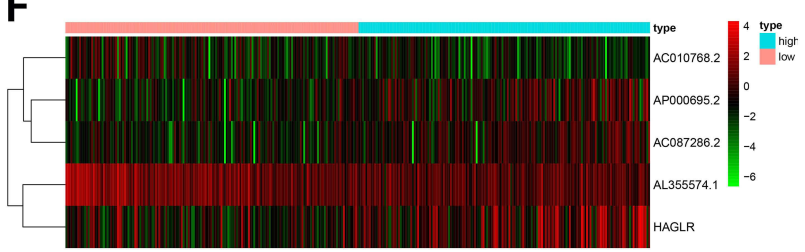

I

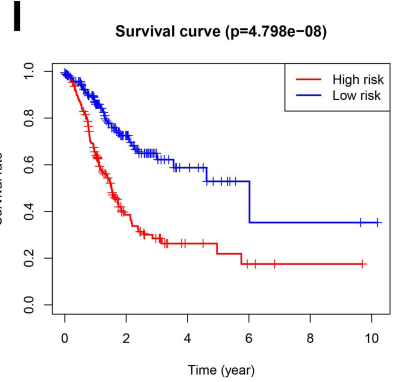

J

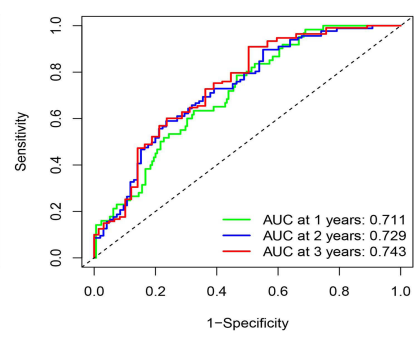

Figure 4 Prognostic analysis of the 5 autophagy-related IncRNAs signature in the test set and the entire set. (A) Distribution of the risk score in the test set. (B) Distribution of the risk score in the entire set. (C) The scatter dot plot of GC patients' survival status in the test set. (D) The scatter dot plot of GC patients' survival status in the entire set. (E) The heatmap of the 5 autophagy-related IncRNAs expression in the test set. (F) The heatmap of the 5 autophagy-related IncRNAs expression in the entire set. (G) Kaplan-Meier survival analysis for patients between the high-risk and low-risk groups in the test set. (H) The ROC for the autophagy-related IncRNAs signature in the test set. (I) Kaplan-Meier survival analysis for patients between the high-risk and low-risk groups in the entire set. (J) The ROC for the autophagy-related IncRNAs signature in the test set.

low-risk group patients was remarkably better than the high-risk group patients for almost all hierarchical cohorts. To sum up, these results suggested that the prognostic signature might serve a pivotal role in determining GC patients' prognosis.

\section{TMB Analysis and Immune Microenvironment Assessment}

We used the R package "maftools" to analyze the mutational data in the entire TCGA set. The top 30 genes were listed to compare the differences of frequent mutational genes in lowrisk and high-risk groups, respectively. According to Figure 8, we found that TTN, TP53, LRP1B, MUC16, SYNE1, ARID1A, CSMD3, and FAT4 were the most frequent mutational genes. Besides, FLG and DNAH5 were part of the top 10 frequent mutational genes in the high-risk group, while PCLO and OBSCN belonged to the top 10 frequent mutational genes in the low-risk group.

We further analyzed the level of 22 infiltrating immune cells in different risk groups. The proportions of B cells memory, T cells follicular helper, and NK cells activated in the low-risk group were much higher than that in the highrisk group. In contrast, the proportion of macrophages M2 in the high-risk group was increased compared with those in the low-risk group. Among these cells, the risk score was negatively correlated with the infiltration of B cells memory $(P=0.007)$ and $\mathrm{T}$ cells follicular helper $(P=$ $0.013)$. In contrast, the risk score was positively associated with the infiltration of macrophages M2 $(P=0.00015$, Figure 9). Furthermore, ssGSEA was performed to evaluate the enrichment levels of immune cells, biological processes, or pathways in GC samples. The results showed 
A

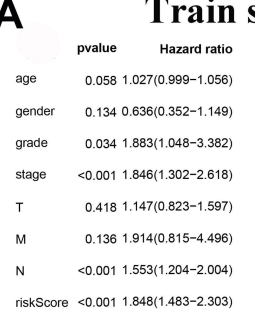

riskScore $<0.0011 .848(1.483-2.303)$
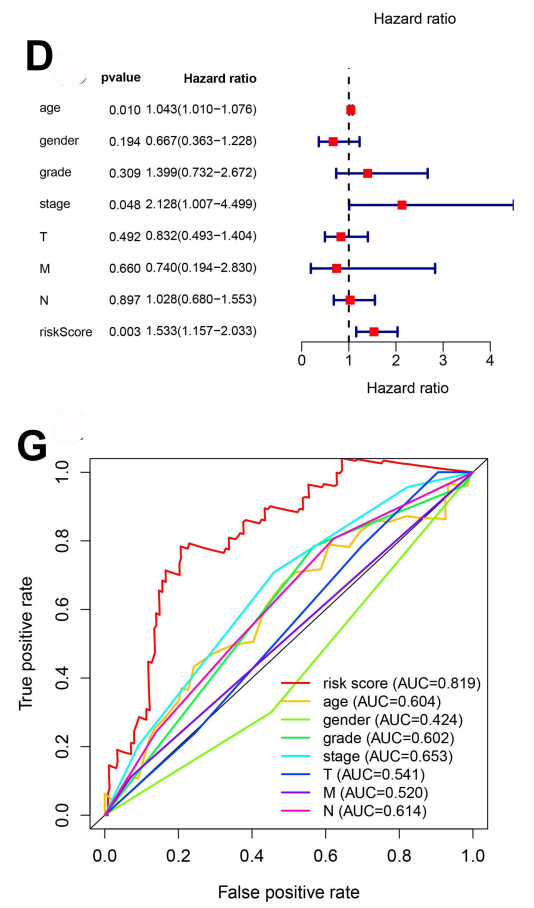

B

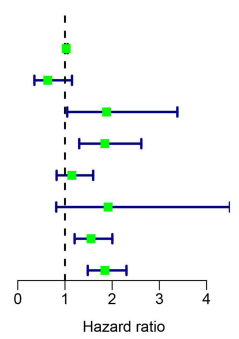

$$
\begin{gathered}
\text { age } \\
\text { gen } \\
\text { grade } \\
\text { staa } \\
\text { T } \\
\text { M } \\
\text { N } \\
\text { risk }
\end{gathered}
$$
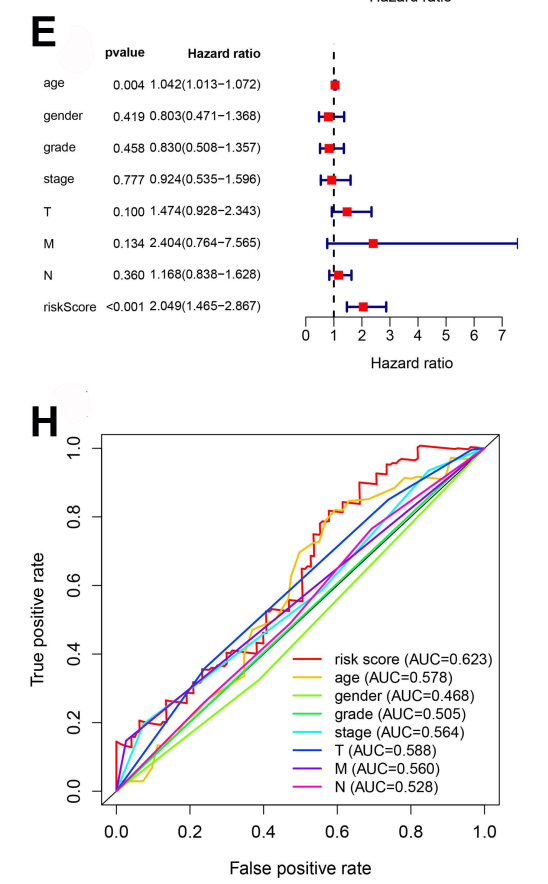
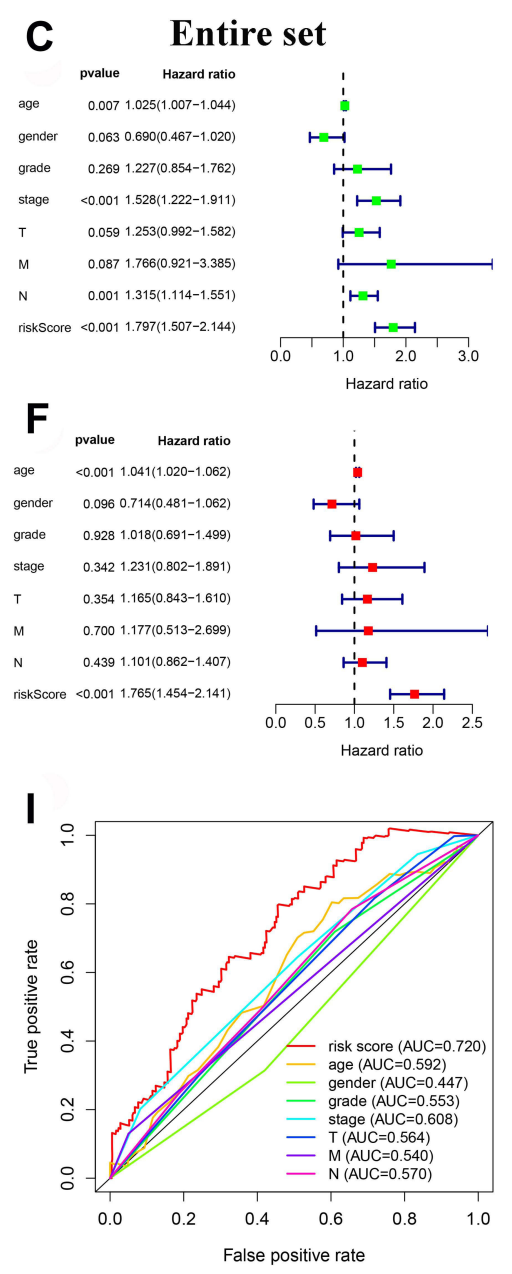

Figure 5 Independent prognostic value of the 5 autophagy-related IncRNAs prognostic signature. (A) The univariate Cox regression analysis of the 5 IncRNAs and clinicopathologic parameters in the train set. (B) The univariate Cox regression analysis of the 5 IncRNAs and clinicopathologic parameters in the test set. (C) The univariate Cox regression analysis of the 5 IncRNAs and clinicopathologic parameters in the entire set. (D) The multivariate Cox regression analysis of the 5 IncRNAs and clinicopathologic parameters in the train set. (E) The multivariate Cox regression analysis of the 5 IncRNAs and clinicopathologic parameters in the test set. (F) The multivariate Cox regression analysis of the 5 IncRNAs and clinicopathologic parameters in the entire set. (G) The ROC analysis of the autophagy-related prognostic signature and clinicopathologic features in the train set. $(\mathbf{H})$ The ROC analysis of the autophagy-related prognostic signature and clinicopathologic features in the test set. $(\mathbf{I})$ The ROC analysis of the autophagy-related prognostic signature and clinicopathologic features in the entire set.

that risk scores were closely correlated with infiltrating immune cell subset (Figure 10).

\section{LncRNA-mRNA Co-Expression Analysis and Functional Enrichment Analysis}

The IncRNA-mRNA co-expression network was constructed to explore the potential mechanisms of 5 IncRNAs from the autophagy-related prognostic signature in GC. As shown in Figure 11A, we observed that these lncRNAs had a significant association with 44 mRNAs (| $\mathrm{R} \mid)>0.3$ and $P<0.001)$. Then, the Sankey diagram revealed the correlation of 5 lncRNAs, 44 mRNAs, and risk types (Figure 11B). Furthermore, according to GO analysis for mRNAs co-expressed with 5 lncRNAs, we found that most enriched terms related to autophagy. The
KEGG analysis also revealed that autophagy was the most significantly enriched pathway (Figure 11C and D).

\section{PCA and GSEA}

PCA was performed to assay the distinct distribution between low- and high-risk groups according to the whole genome, autophagy-related lncRNAs, and 5 lncRNAs from the autophagy-related prognostic signature (Figure 12). The results indicated that GC patients were divided into two sections based on 5 IncRNAs from the autophagy-related prognostic signature. The autophagy status of patients in the low-risk group was also different from those in the high-risk group. Besides, GSEA was performed to explore whether the autophagy pathway was enriched in high-risk groups. The results showed 


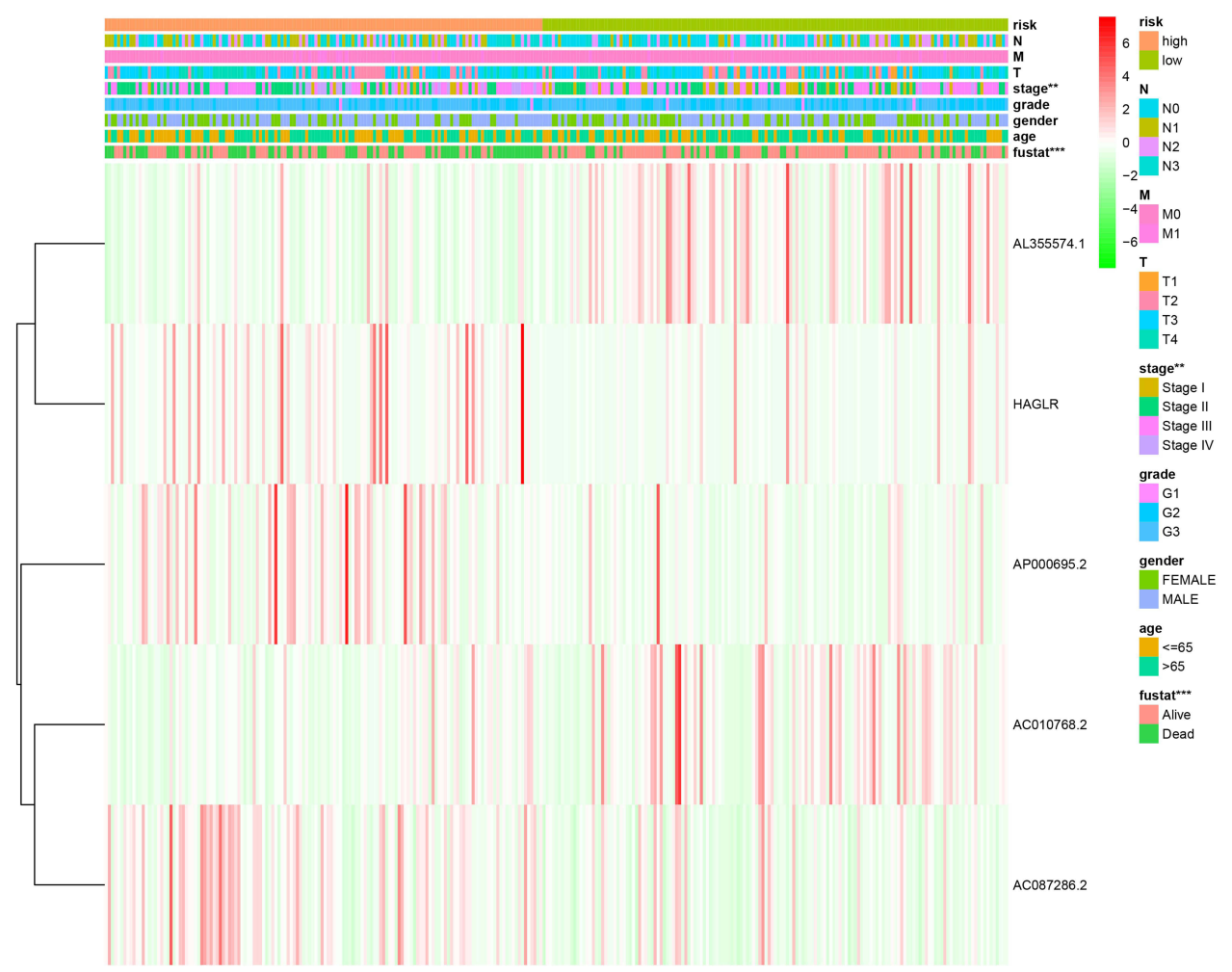

Figure 6 The relationship between the risk score from the autophagy-related IncRNA prognostic signature and clinicopathological features. Notes: $* * p<0.01$, $* * * p<0.001$.

that GC patients in high-risk groups were mainly involved in pathways related to autophagy and immune processes, including Hedgehog (Hh) signaling pathway and MAPK signaling pathway (Figure 13).

\section{Discussion}

As the most common malignant tumor of the digestive system, gastric cancer is still a challenge for clinicians and researchers in risk stratification and prognosis prediction. The study of the autophagy mechanism opens up a novel perspective for GC. More and more researches have shown that autophagy participates in GC progression and prognosis. ${ }^{12-14}$ Therefore, autophagy-related biomarkers may play a crucial role in predicting cancer risk and survival outcomes of GC patients. Moreover, recent studies have revealed that lncRNAs might be involved in autophagy-inducing progression or inhibition of several types of tumors. ${ }^{20,22,23}$ For example, Wu et al discovered that lncRNA SNHG11 facilitates the progression of GC via activating oncogenic autophagy and the wnt/ $\beta$-catenin pathway. ${ }^{24}$ Wang et al revealed that IncRNA MALAT1 promotes GC progression by suppressing autophagic flux and converting fibroblasts to CAFs. ${ }^{25}$ Yang et al also found that lncRNA CCAT1 enhances autophagy and facilitates the development of GC via the miR-140-3p/ATG5 axis. ${ }^{26}$ However, most studies focused on the role of single lncRNA related to autophagy in the progression and prognosis of GC. Based on the above, it is of great significance to investigate the prognostic value of autophagy-related lncRNA signature and its role in the TIME of GC.

In this study, we first obtained 17 autophagy-related IncRNAs significantly associated with GC patients' survival through univariate Cox regression analysis. Then, we identified 5 autophagy-related lncRNAs (AL355574.1, AC010768.2, AP000695.2, AC087286.2, and HAGLR) to construct a prognostic signature in the train set by multivariate Cox regression analysis. Based on the 5 autophagy-related lncRNA prognostic signature, the risk score of each GC patient could be calculated and used to predict the survival of GC patients. The survival of GC patients with high-risk scores was worse than those with low-risk scores. ROC curve analysis further confirmed that the prognostic signature could be used to predict GC patients' survival reliably. Furthermore, we discovered that the risk score was positively associated with tumor stage and survival state. The autophagy-related lncRNA 

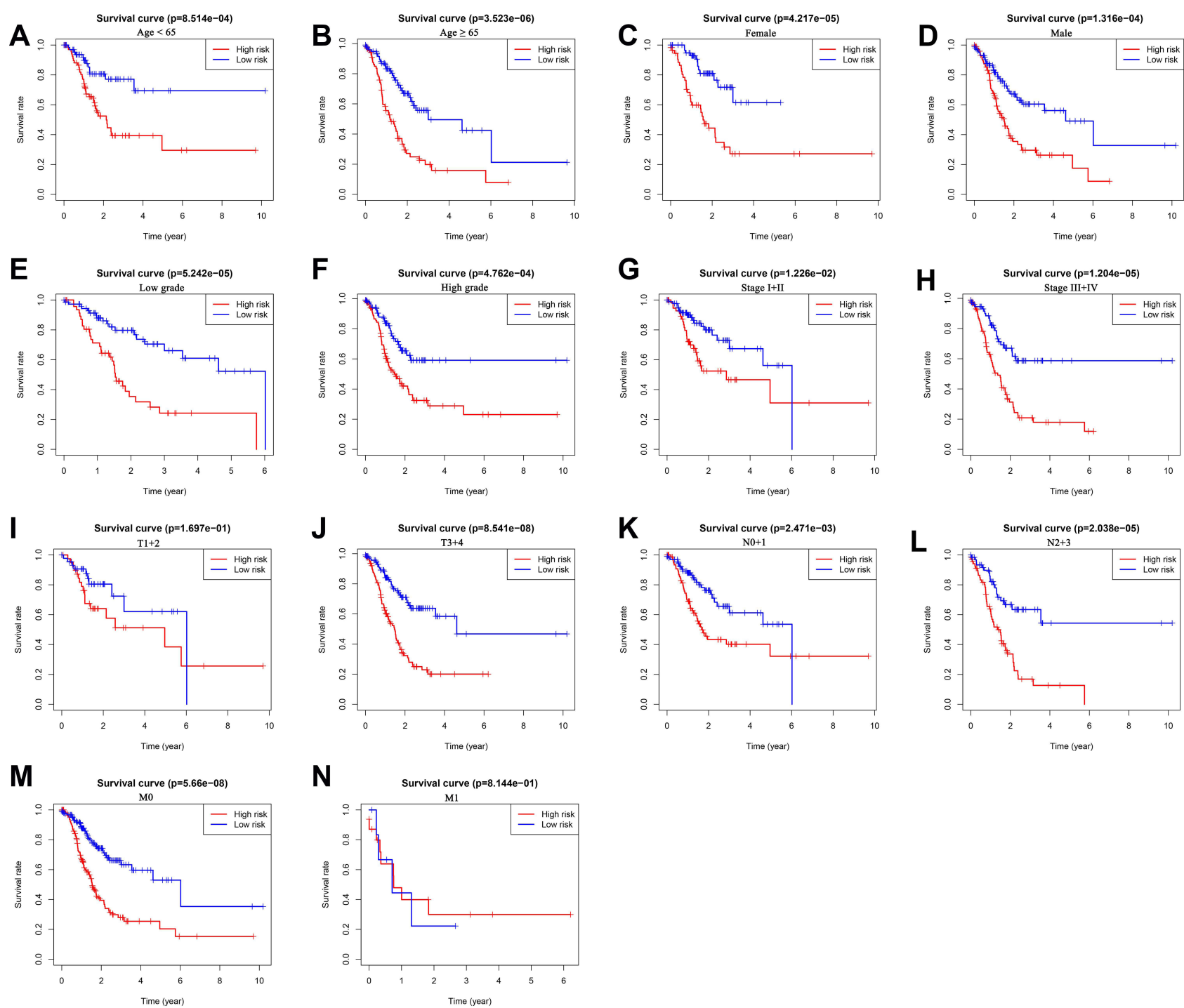

Figure 7 The survival differences between high- and low-risk GC patients stratified by clinicopathological characteristics. (A-N) The difference in overall survival stratified by age (<65 and $\geq 65$ ), gender (female and male), grade (low grade and high grade), tumor stage (stage I+II and stage III+IV), T stage (TI+2 and T3+4), N stage (N0+I and $\mathrm{N} 2+3)$, $M$ stage $(\mathrm{MO}, \mathrm{MI})$ between two groups.
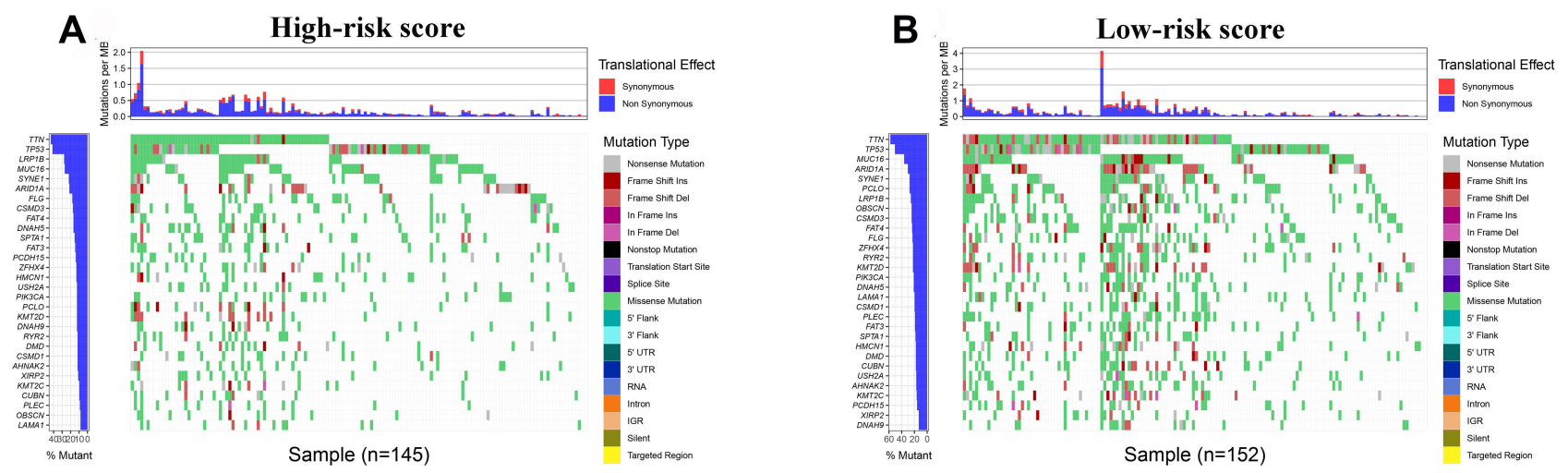

Figure 8 Tumor mutational burden analysis. (A and $\mathbf{B}$ ) The top 30 mutational genes in the high-risk and low-risk groups. 
A
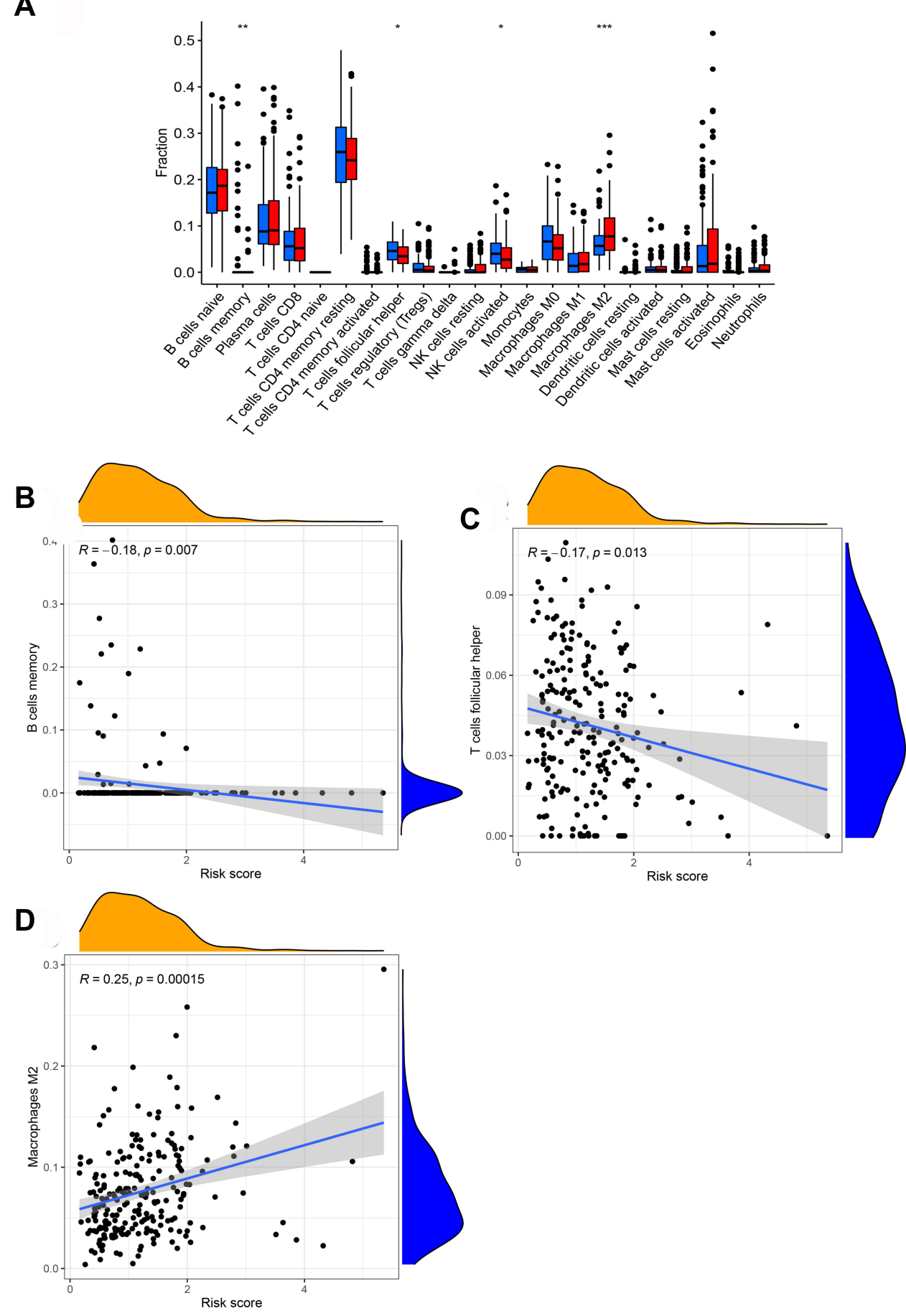

Figure 9 The association between the risk score and infiltrating immune cells. (A) The ratio differentiation of infiltrating immune cells between the high-risk and low-risk groups. (B-D) 3 kinds of infiltrating immune cells associated with the risk score.

Notes: $* p<0.05, * * p<0.01, * * * p<0.001$.

signature also performed well in the test set and the entire set. Therefore, we speculated that the 5 autophagy-related IncRNAs prognostic signature was significantly correlated with the development and prognosis of GC and could be a powerful indicator of the clinical outcome of GC patients.
So far, among 5 autophagy-related lncRNAs, only HAGLR has been reported to be involved in cancer. HOXD antisense growth-associated long non-coding RNA (HAGLR, also known as LncRNA HOXD-AS1) is an evolutionarily conserved transcript of the HOXD gene 
A

Risk 官 low 官 high

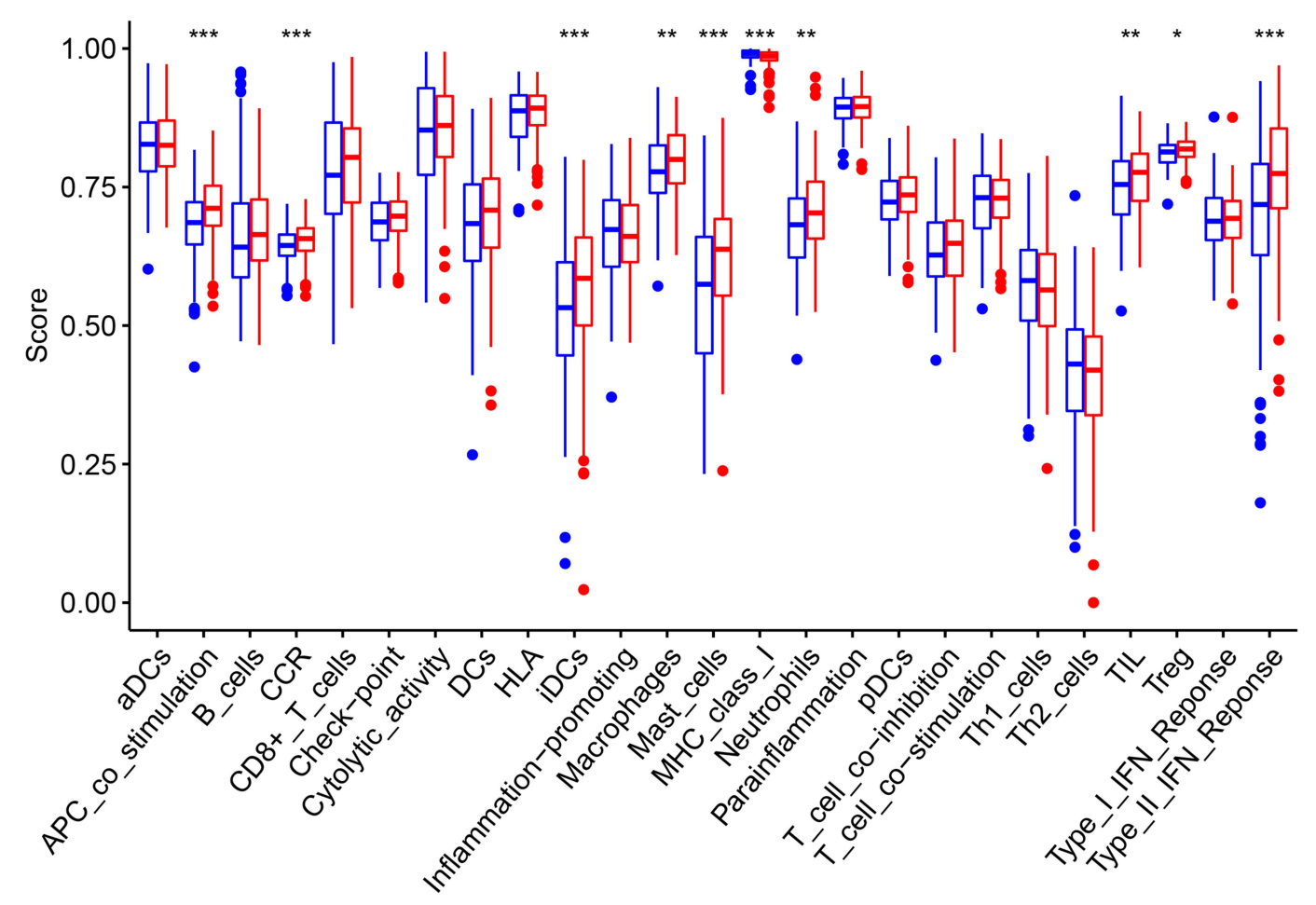

B

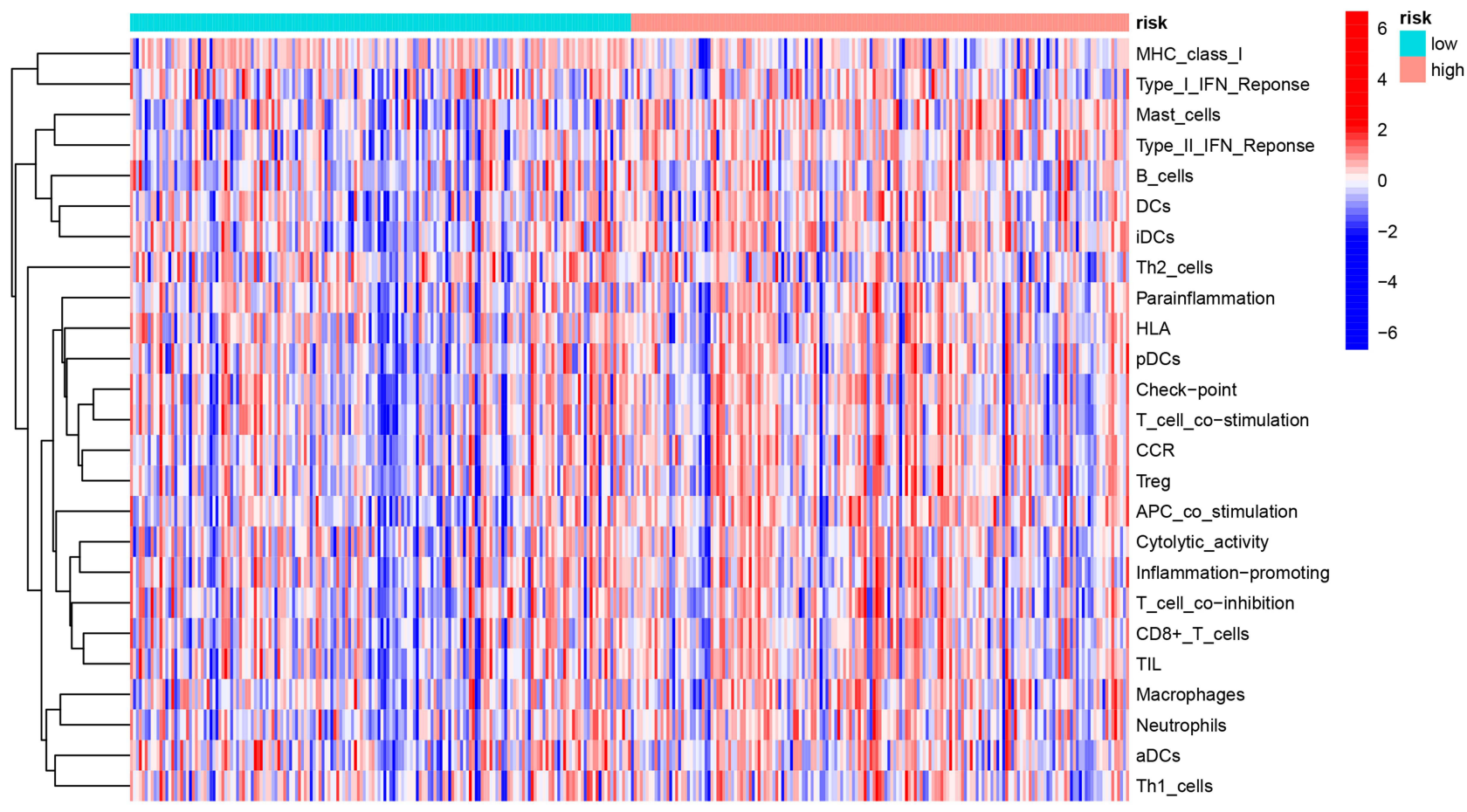

Figure 10 The correlation between the risk score and the characterization of tumor immune microenvironment. (A) The value of 25 immune-related signatures between the high-risk and low-risk groups. (B) The heatmap of 25 immune-related signatures. $* P<0.05, * * P<0.0$ I, $* * * P<0.00$ I.

cluster located on chromosome $2 \mathrm{q} 31.2 .^{27,28}$ Several studies have shown that HAGLR is associated with the proliferation, invasion, and metastasis of tumor cells. ${ }^{29-34}$
Besides, the prognostic value of HAGLR in tumors has been evaluated. For example, Dong et al revealed that high expression of HAGLR is positively correlated with poorer 


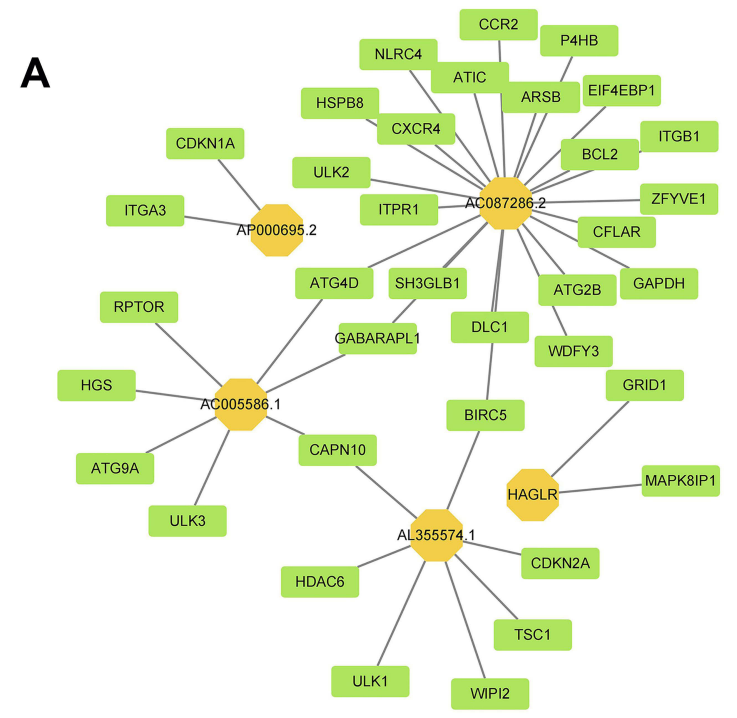

B

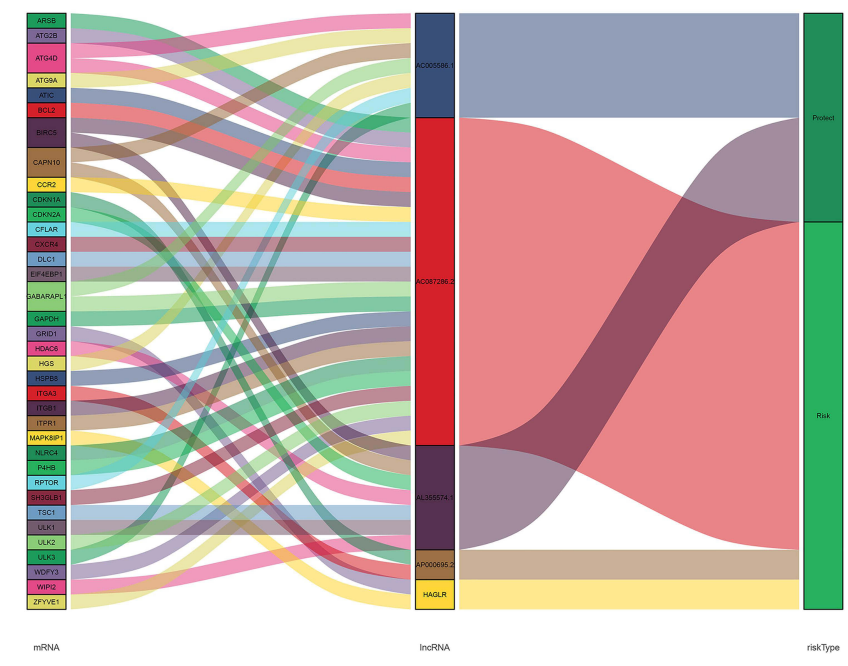

C

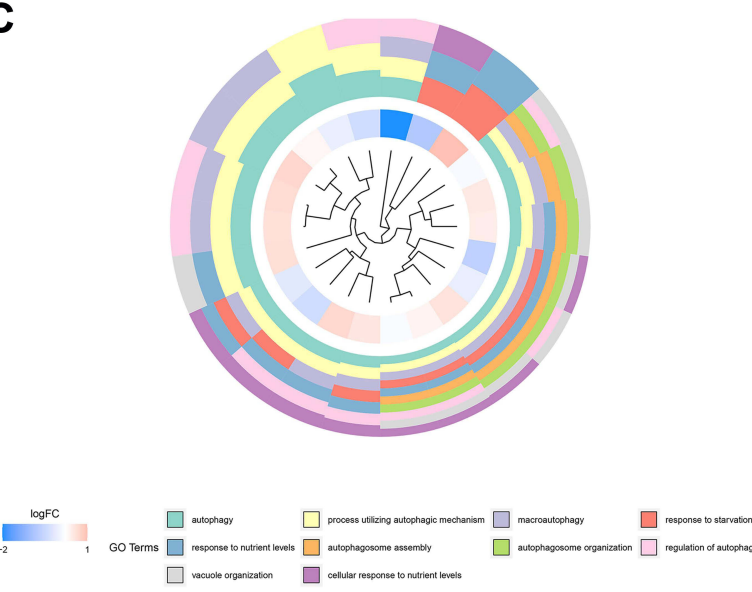

D

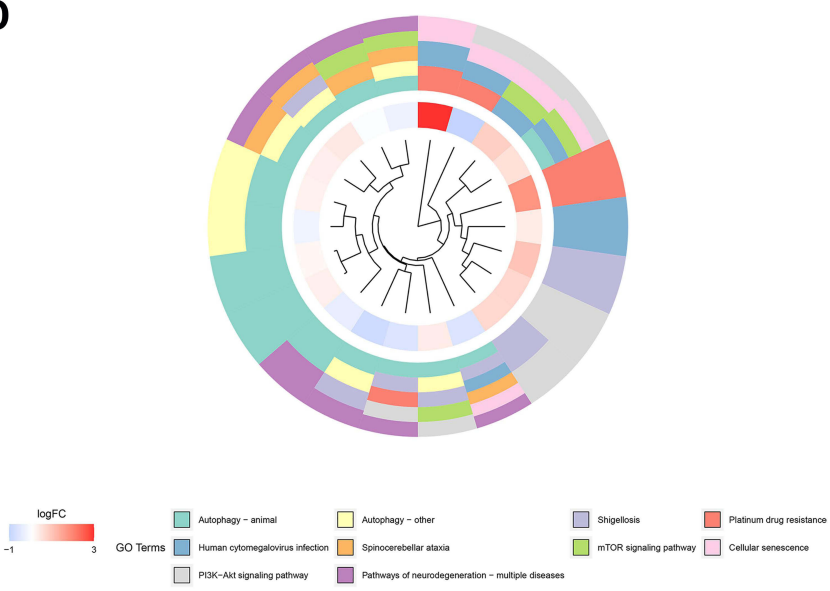

Figure II LncRNA-mRNA co-expression network and functional enrichment analysis. (A) Construction of the IncRNA-mRNA co-expression network based on 5 autophagy-related IncRNAs. (B) A Sankey diagram showed the correlation of IncRNAs, mRNAs, and risk type. (C) GO analysis of the mRNAs co-expressed with 5 autophagy-related IncRNAs. (D) KEGG analysis of the mRNAs co-expressed with 5 autophagy-related IncRNAs.

A

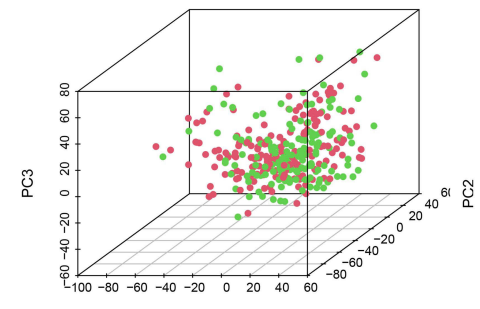

PC1
B

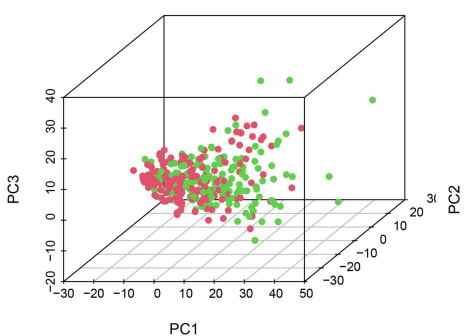

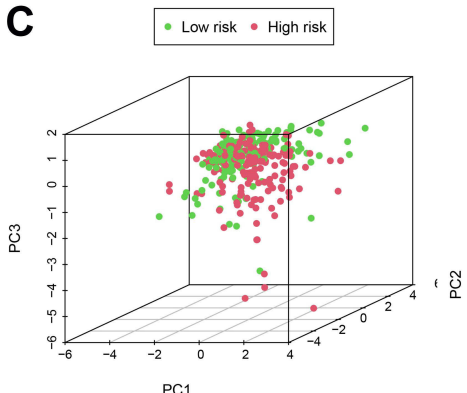

PC1

Figure 12 The differences and similarities among grouped samples. (A) PCA of the whole-genome expression profiles. (B) PCA of autophagy-related IncRNA set. (C) PCA of the 5 autophagy-related IncRNA prognostic signature.

prognosis of epithelial ovarian cancer patients. ${ }^{28} \mathrm{Li}$ et al also indicated that increased expression of HOXD-AS1 is related to aggressive tumor phenotype and poor prognosis in colorectal cancer. ${ }^{35}$ Although the role of these 5 lncRNAs in GC has not been reported in previous studies, we should not underestimate their importance. We further 

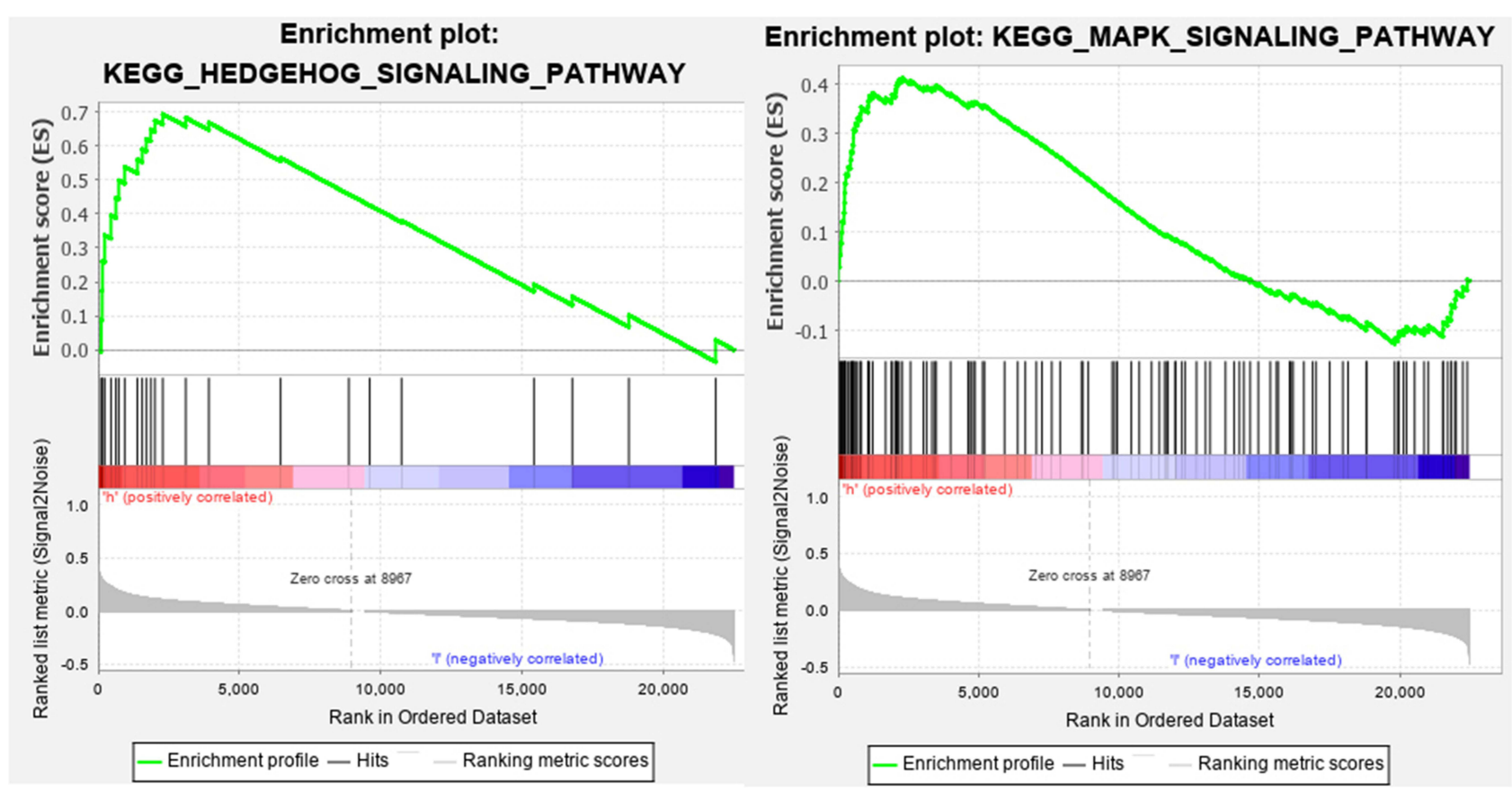

Figure 13 GSEA of GC patients based on the autophagy-related IncRNA prognostic signature.

compared the immune cell content of low- and high-risk groups to investigate the relationship between the autophagy signature and immune cell infiltration. The results showed that the proportions of B cells memory, T cells follicular helper, macrophages M2, and NK cells were significantly different in the two risk groups. Among these cells, the risk score was negatively correlated with the infiltration of $\mathrm{B}$ cells memory and $\mathrm{T}$ cells follicular helper. In contrast, the risk score was positively associated with the infiltration of macrophages M2. Our study suggested that the autophagy signature had an impact on the TIME of GC.

Furthermore, GSEA showed that GC patients with high-risk scores were enriched in pathways related to autophagy and immune processes, including the $\mathrm{Hh}$ signaling pathway and MAPK signaling pathway. Aberrant activation of Hh signaling is related to multiple gastrointestinal diseases, such as gut malrotation, Pallister-Hall syndrome, and GC. ${ }^{36}$ Aberrant Hh pathway activation can promote metastasis, tumor immune tolerance, and drug resistance of GC cells. ${ }^{37}$ In gastritis, Hh signaling is required for polarizing myeloid cells into myeloid-derived suppressor cells, which contribute to evading immune surveillance, suggesting the crucial role of Hh signaling in the development of cancer. ${ }^{38,39}$ Furthermore, several studies have shown that Hh signaling pathway interactive with autophagy in GC. For example, Tang et al demonstrated the activation of autophagy through the inhibition of Hh signaling in several cancer cell lines, including MKN45 (gastric cancer cells), ES2 (ovarian cancer cells), HT29 (colon cancer cells), and $\mathrm{H} 4$ (glioma cells). ${ }^{40}$ Won et al also suggested that autophagy might be correlated with $\mathrm{Hh}$ signaling pathway in GC. ${ }^{41}$ However, the underlying molecular mechanisms remain to delineate. The MAPK pathway, as one of the most ancient signal transduction pathways, also plays a crucial role in regulating cell proliferation, autophagy, and immune response. ${ }^{42}$ The MAPK pathway mainly consists of the extracellular signal regulated kinase (ERK), the c-jun N-terminal kinase (JNK), and the p38 MAPK ${ }^{43}$ H. pylori, as an oncogenic factor of GC, could secrete JHP0290 to induce TNF $\alpha$ transcription through the activation of ERK, leading to immunosurveillance escape. ${ }^{44}$ JNK activation regulates autophagy via two distinct mechanisms: on the one hand, it stimulates autophagy through promoting Bcl-2/Bcl-xL phosphorylation; ${ }^{45}$ on the other hand, it contributes to autophagy induction through transactivation of target genes such as Sestrin 2 and DRAM. $^{46,47}$ These findings indicated that these 5 lncRNAs might modulate autophagy and immune response through the above pathway, resulting in differences in survival outcomes between high-risk and lowrisk groups. 


\section{Conclusion}

In summary, we constructed a 5 autophagy-related lncRNA prognostic signature (AL355574.1, AC010768.2, AP000695.2, AC087286.2, and HAGLR) by Cox regression analysis. This signature could be an independent prognostic indicator for GC patients and has a higher prediction efficiency than other clinicopathological parameters. Furthermore, we discovered the crucial role of autophagy-related lncRNA signature in TIME of GC. Our study suggests that the 5 autophagy-related lncRNA prognostic signature could be a powerful indicator of GC patients' clinical outcome and might provide promising targets for immunotherapy. However, future studies are needed to validate our prognostic signature and to investigate the mechanisms of these autophagy-related lncRNAs in modulating TIME.

\section{Acknowledgments}

We gratefully acknowledge the TCGA Research Network for the availability of data in this study.

\section{Author Contributions}

All authors made substantial contributions to conception and design, acquisition of data, or analysis and interpretation of data; took part in drafting the article or revising it critically for important intellectual content; agreed to submit to the current journal; gave final approval of the version to be published; and agree to be accountable for all aspects of the work.

\section{Funding}

This study was supported by the National Key Research and Development Program of China (2018YFC2002000).

\section{Disclosure}

The authors report no conflicts of interest in this work.

\section{References}

1. Sung H, Ferlay J, Siegel RL, et al. Global Cancer Statistics 2020: GLOBOCAN estimates of incidence and mortality worldwide for 36 cancers in 185 countries. CA Cancer J Clin. 2021;71(3):209-249. doi: $10.3322 /$ caac. 21660

2. Smyth EC, Nilsson M, Grabsch HI, van Grieken NC, Lordick F. Gastric cancer. Lancet. 2020;396(10251):635-648. doi:10.1016/ S0140-6736(20)31288-5

3. Addeo M, Di Paola G, Verma HK, et al. Gastric cancer stem cells: a glimpse on metabolic reprogramming. Front Oncol. 2021;11:698394. doi:10.3389/fonc.2021.698394
4. Verma HK, Falco G, Bhaskar L. Molecular signaling pathways involved in gastric cancer chemoresistance. In: Theranostics Approaches to Gastric and Colon Cancer. Springer; 2020:117-134.

5. Fu M, Gu J, Jiang P, Qian H, Xu W, Zhang X. Exosomes in gastric cancer: roles, mechanisms, and applications. Mol Cancer. 2019;18 (1):41. doi:10.1186/s12943-019-1001-7

6. Ji X, Bu ZD, Yan Y, et al. The 8th edition of the American Joint Committee on Cancer tumor-node-metastasis staging system for gastric cancer is superior to the 7th edition: results from a Chinese mono-institutional study of 1663 patients. Gastric Cancer. 2018;21 (4):643-652. doi:10.1007/s10120-017-0779-5

7. White E. The role for autophagy in cancer. $J$ Clin Invest. 2015;125 (1):42-46. doi:10.1172/JCI73941

8. Kimmelman AC, White E. Autophagy and tumor metabolism. Cell Metab. 2017;25(5):1037-1043. doi:10.1016/j.cmet.2017.04.004

9. Nah J, Yuan J, Jung YK. Autophagy in neurodegenerative diseases: from mechanism to therapeutic approach. Mol Cells. 2015;38 (5):381-389. doi:10.14348/molcells.2015.0034

10. Amaravadi RK, Kimmelman AC, Debnath J. Targeting autophagy in cancer: recent advances and future directions. Cancer Discov. 2019;9 (9):1167-1181. doi:10.1158/2159-8290.CD-19-0292

11. Zhong Z, Sanchez-Lopez E, Karin M. Autophagy, inflammation, and immunity: a Troika governing cancer and its treatment. Cell. 2016;166(2):288-298. doi:10.1016/j.cell.2016.05.051

12. Zhang Q, Wu S, Zhu J, Chai D, Gan H. Down-regulation of ASIC1 suppressed gastric cancer via inhibiting autophagy. Gene. 2017;608:79-85. doi:10.1016/j.gene.2017.01.014

13. Qiu J, Sun M, Wang Y, Chen B. Identification and validation of an individualized autophagy-clinical prognostic index in gastric cancer patients. Cancer Cell Int. 2020;20:178. doi:10.1186/s12935-02001267-y

14. Rinninella E, Cintoni M, Raoul P, et al. Muscle mass, assessed at diagnosis by L3-CT scan as a prognostic marker of clinical outcomes in patients with gastric cancer: a systematic review and meta-analysis Clin Nutr. 2020;39(7):2045-2054. doi:10.1016/j.clnu.2019.10.021

15. Spizzo R, Almeida MI, Colombatti A, Calin GA. Long non-coding RNAs and cancer: a new frontier of translational research? Oncogene. 2012;31(43):4577-4587. doi:10.1038/onc.2011.621

16. Nagano T, Fraser P. No-nonsense functions for long noncoding RNAs. Cell. 2011;145(2):178-181. doi:10.1016/j.cell.2011.03.014

17. Fabbri M, Girnita L, Varani G, Calin GA. Decrypting noncoding RNA interactions, structures, and functional networks. Genome Res. 2019;29(9):1377-1388. doi:10.1101/gr.247239.118

18. Slack FJ, Chinnaiyan AM. The role of non-coding RNAs in oncology. Cell. 2019;179(5):1033-1055. doi:10.1016/j.cell.2019.10.017

19. Sexton RE, Al Hallak MN, Diab M, Azmi AS. Gastric cancer: a comprehensive review of current and future treatment strategies. Cancer Metastasis Rev. 2020;39(4):1179-1203.

20. Wu Q, Ma J, Wei J, Meng W, Wang Y, Shi M. IncRNA SNHG11 promotes gastric cancer progression by activating the Wnt/beta-catenin pathway and oncogenic autophagy. Mol Ther. 2021;29:12581278

21. Chen JF, Wu P, Xia R, et al. STAT3-induced lncRNA HAGLROS overexpression contributes to the malignant progression of gastric cancer cells via mTOR signal-mediated inhibition of autophagy. Mol Cancer. 2018;17(1):6. doi:10.1186/s12943-017-0756-y

22. Xiong H, Ni Z, He J, et al. LncRNA HULC triggers autophagy via stabilizing Sirt1 and attenuates the chemosensitivity of HCC cells. Oncogene. 2017;36(25):3528-3540. doi:10.1038/onc.2016.521

23. Liu X, Xiao ZD, Han L, et al. LncRNA NBR2 engages a metabolic checkpoint by regulating AMPK under energy stress. Nat Cell Biol. 2016;18(4):431-442. doi:10.1038/ncb3328

24. Wu Q, Ma J, Wei J, Meng W, Wang Y, Shi M. lncRNA SNHG11 promotes gastric cancer progression by activating the wnt/beta-catenin pathway and oncogenic autophagy. Mol Ther. 2021;29(3):12581278. doi:10.1016/j.ymthe.2020.10.011 
25. Wang $Z$, Wang $X$, Zhang $T$, et al. LncRNA MALAT1 promotes gastric cancer progression via inhibiting autophagic flux and inducing fibroblast activation. Cell Death Dis. 2021;12(4):368. doi:10.1038/ s41419-021-03645-4

26. Yang F, Peng ZX, Ji WD, et al. LncRNA CCAT1 upregulates ATG5 to enhance autophagy and promote gastric cancer development by absorbing miR-140-3p. Dig Dis Sci. 2021. doi:10.1007/s10620-021-07187-9

27. Gu P, Chen X, Xie R, et al. IncRNA HOXD-AS1 regulates proliferation and chemo-resistance of castration-resistant prostate cancer via recruiting WDR5. Mol Ther. 2017;25(8):1959-1973. doi:10.1016/j. ymthe.2017.04.016

28. Dong S, Wang R, Wang H, et al. HOXD-AS1 promotes the epithelial to mesenchymal transition of ovarian cancer cells by regulating miR186-5p and PIK3R3. J Exp Clin Cancer Res. 2019;38(1):110. doi:10.1186/s13046-019-1103-5

29. Chen S, Li K. HOXD-AS1 facilitates cell migration and invasion as an oncogenic lncRNA by competitively binding to miR-877-3p and upregulating FGF2 in human cervical cancer. BMC Cancer. 2020;20 (1):924. doi:10.1186/s12885-020-07441-9

30. Yang MH, Zhao L, Wang L, et al. Nuclear lncRNA HOXD-AS1 suppresses colorectal carcinoma growth and metastasis via inhibiting HOXD3-induced integrin beta3 transcriptional activating and MAPK/ AKT signalling. Mol Cancer. 2019;18(1):31. doi:10.1186/s12943019-0955-9

31. Chen Y, Zhao F, Cui D, et al. HOXD-AS1/miR-130a sponge regulates glioma development by targeting E2F8. Int J Cancer. 2018;142 (11):2313-2322. doi:10.1002/ijc.31262

32. Yang C, Shen S, Zheng X, et al. Long noncoding RNA HAGLR acts as a microRNA-143-5p sponge to regulate epithelial-mesenchymal transition and metastatic potential in esophageal cancer by regulating LAMP3. FASEB J. 2019;33(9):10490-10504. doi:10.1096/ fj.201802543RR

33. Wang H, Huo X, Yang XR, et al. STAT3-mediated upregulation of IncRNA HOXD-AS1 as a ceRNA facilitates liver cancer metastasis by regulating SOX4. Mol Cancer. 2017;16(1):136. doi:10.1186/ s12943-017-0680-1

34. Wang Y, Zhang W, Wang Y, Wang S. HOXD-AS1 promotes cell proliferation, migration and invasion through miR-608/FZD4 axis in ovarian cancer. Am J Cancer Res. 2018;8(1):170-182.

35. Li X, Zhao X, Yang B, et al. Long non-coding RNA HOXD-AS1 promotes tumor progression and predicts poor prognosis in colorectal cancer. Int J Oncol. 2018;53(1):21-32.

36. Kolterud A, Grosse AS, Zacharias WJ, et al. Paracrine Hedgehog signaling in stomach and intestine: new roles for hedgehog in gastrointestinal patterning. Gastroenterology. 2009;137(2):618-628. doi:10.1053/j.gastro.2009.05.002
37. Konstantinou D, Bertaux-Skeirik N, Zavros Y. Hedgehog signaling in the stomach. Curr Opin Pharmacol. 2016;31:76-82. doi:10.1016/j. coph.2016.09.003

38. Ding L, Hayes MM, Photenhauer A, et al. Schlafen 4-expressing myeloid-derived suppressor cells are induced during murine gastric metaplasia. J Clin Invest. 2016;126(8):2867-2880. doi:10.1172/ JCI82529

39. Merchant JL, Ding L. Hedgehog signaling links chronic inflammation to gastric cancer precursor lesions. Cell Mol Gastroenterol Hepatol. 2017;3(2):201-210. doi:10.1016/j.jcmgh.2017.01.004

40. Tang X, Deng L, Chen Q, et al. Inhibition of Hedgehog signaling pathway impedes cancer cell proliferation by promotion of autophagy. Eur $J$ Cell Biol. 2015;94(5):223-233. doi:10.1016/j. ejcb.2015.03.003

41. Won KY, Kim GY, Lim SJ, et al. Autophagy is related to the hedgehog signaling pathway in human gastric adenocarcinoma: prognostic significance of Beclin-1 and Gli2 expression in human gastric adenocarcinoma. Pathol Res Pract. 2015;211(4):308-315. doi:10.1016/j. prp.2014.11.005

42. Cargnello M, Roux PP. Activation and function of the MAPKs and their substrates, the MAPK-activated protein kinases. Microbiol Mol Biol Rev. 2011;75(1):50-83.

43. Dong C, Davis RJ, Flavell RA. MAP kinases in the immune response. Annu Rev Immunol. 2002;20:55-72. doi:10.1146/annurev. immunol.20.091301.131133

44. Pathak SK, Tavares R, de Klerk N, Spetz AL, Jonsson AB. Helicobacter pylori protein JHP0290 binds to multiple cell types and induces macrophage apoptosis via tumor necrosis factor (TNF)dependent and independent pathways. PLoS One. 2013;8(11):e77872. doi:10.1371/journal.pone.0077872

45. Zhou F, Yang Y, Xing D. Bcl-2 and Bcl-xL play important roles in the crosstalk between autophagy and apoptosis. FEBS J. 2011;278 (3):403-413. doi:10.1111/j.1742-4658.2010.07965.x

46. Zhang XY, Wu XQ, Deng R, Sun T, Feng GK, Zhu XF. Upregulation of sestrin 2 expression via JNK pathway activation contributes to autophagy induction in cancer cells. Cell Signal. 2013;25(1):150158. doi:10.1016/j.cellsig.2012.09.004

47. Lorin S, Borges A, Ribeiro Dos Santos L, et al. c-Jun NH2-terminal kinase activation is essential for DRAM-dependent induction of autophagy and apoptosis in 2-methoxyestradiol-treated Ewing sarcoma cells. Cancer Res. 2009;69(17):6924-6931. doi:10.1158/00085472.CAN-09-1270
International Journal of General Medicine

\section{Publish your work in this journal}

The International Journal of General Medicine is an international, peer-reviewed open-access journal that focuses on general and internal medicine, pathogenesis, epidemiology, diagnosis, monitoring and treatment protocols. The journal is characterized by the rapid reporting of reviews, original research and clinical studies across all disease areas. The manuscript management system is completely online and includes a very quick and fair peer-review system, which is all easy to use. Visit http://www.dovepress.com testimonials.php to read real quotes from published authors. 